\title{
Harnessing B cells for cancer immunotherapy.
}

\author{
Yanqiu Li, Shala Yuan and Jiusheng Deng* \\ Department of Hematology and Medical Oncology, Winship Cancer Institute, Emory University, Atlanta, USA.
}

\begin{abstract}
$B$ cells are a heterogeneous population in immune defense system with multidirectional functions. In cancer patients, B cell infiltrates are associated with a significant increase of overall survival. We have recently developed a GM-CSF and IL-4 derived fusion cytokine named GIFT4, which has the capability to prime naïve B cells into anti-tumor immune effector cells. Herein, we overview current research findings on B cell anti-tumor functions and B cell-based approaches for cancer immunotherapy. We predict that GIFT4augmented $B$ cells as a potent cellular therapeutic could provide a new approach for cancer immunotherapy.
\end{abstract}

Keywords: B cells, Tumor microenvironment, GIFT4 fusokine, Cancer immunotherapy.

Accepted July 25, 2016

\section{Introduction}

\section{B Cell Functions}

$\mathrm{B}$ cells are one of the two adaptive arms partnering with $\mathrm{T}$ cells in immune defense system against infections by virus, bacteria, fungi and parasites [1]. Originated from hematopoietic stem cells in bone marrow, B cells contain multiple subsets including antibody-secreting cells, antigen-presenting cells (APC), innate B effector cells and regulatory $\mathrm{B}$ cells [2]. As a heterogeneous population, $\mathrm{B}$ cells possess multidirectional immune functions. For instance, B cells can produce antigen-specific antibodies in response to infectious pathogens or sterile self-antigens [3]. B cells can also present pathogen-derived antigens to $\mathrm{T}$ cells during infections [4]. B effector cells can further produce a variety of immune-stimulatory cytokines such as IL-1, IL-6, IL-12 [5], Granulocyte-macrophage colonystimulating factor (GM-CSF) [6], augment immune response against infections or promote inflammation in autoimmune diseases [6,7]. In contrast, regulatory B cells secrete immune-suppressive cytokines including IL-10 and transforming growth factor- $\beta$ (TGF- $\beta$ ) to attenuate proinflammatory immune response [8]. Emerging evidences show that $\mathrm{B}$ cells also have anti-tumor function $[9,10]$. In preclinical animal model, B cells are required for the successful combined antibody-immunotherapy against murine mesotheliomas [11]. In patients with malignancies, $\mathrm{B}$ cells are also found to correlate with a significant increase of overall survival, and higher number of B cell infiltrates lead to better prognosis [12]. However, B cells in particular regulatory B cells can also act as immunesuppressive cells and facilitate tumor immune escape
$[13,14]$. The dual functional faces of $B$ cells on tumors are likely due to the different $\mathrm{B}$ cell subpopulations, which have distinguished phenotypes and secretomes that either inhibit tumor growth or facilitate malignancy [9].

\section{B Cells in Tumor Microenvironment}

$\mathrm{B}$ cells as well as T cells, natural killer cells, monocytes and other immune cells can infiltrate into tumor microenvironment, distributing from the tumor margin to the tumor core. In patients with tongue squamous cell carcinoma, infiltrated B cells are commonly found in the carcinoma stroma with tumor-suppressive effect [12]. In pancreatic ductal adenocarcinoma, human B cells reside in tertiary lymphoid tissue with two distinct infiltrating patterns: scattered or organized $[15,16]$. High density of organized infiltrating B cells predicts longer survival for patients; highlighting B cells are essential effector cells in the tumor microenvironment of human pancreatic ductal adenocarcinoma [16]. In bladder cancer, human $\mathrm{CD} 20^{+} \mathrm{B}$ cells preferentially migrate into the lamina propria area, and have positive correlation with $\mathrm{T}$ cell infiltration [17]. Further analyses demonstrated that B cell infiltrates have no link with Foxp3 positive regulatory $\mathrm{T}$ cells in tumor microenvironment [17]. Moreover, tertiary lymphoid structures with aggregating $\mathrm{B}$ cells are associated with lung cancer prognosis [18]. In patients with gastric cancer, B cells abundantly infiltrate and aggregate in the gastric cancer stromal microenvironment, accompanied with infiltrated $\mathrm{T}-$ bet $^{+} \mathrm{T}$ cells to form a tertiary lymphoid structure surrounding the tumor [19]. Tumor-associated B cells in gastric cancer microenvironment are proliferating and express Ki67. Importantly, infiltrated B cell number 
is positively linked to relapse-free survival, and B-cell gene expression is significantly connected with improved outcome [19]. It is found that tumor-infiltrating B cells have beneficial effects on prognosis in patients with tongue squamous cell carcinoma [12], pancreatic adenocarcinoma [16], gastric cancer [19], cutaneous melanoma [20,21], breast cancer [22], ovarian cancer [23] and colorectal cancer [24]. However, the mechanisms by which B cells accumulate in the tumor microenvironment and result in better prognosis are not fully understood. One explanation is that tumor-infiltrating B cells express antigen-presentation molecules and function as professional APC to orchestrate T cell-mediated anticancer immunity $[25,26]$. Another reason could be that infiltrated B cells have potent capacity to produce antitumor antigen-specific antibodies, since $\mathrm{CD} 138^{+}$and immunoglobulin kappa C-positive plasma cells have positive impact on anti-tumor immunity and are related to favorable prognosis in cancer patients [24,27]. MUC1 (The polymorphic epithelial mucin) is one of the most specific tumor-associated antigens in human cancers $[24,28]$. Anti-MUC1 IgG antibodies but not IgM in patients are significantly related to better prognosis [22]. Consistently, high density of plasma cells was found surrounding the tertiary lymphoid structures and correlated to $\mathrm{T}$ cell cytotoxicity [29]. Infiltrating B cells can also undergo somatic mutation, clonal expansion, intraclonal variation and isotype switching, eliciting humoral immunity against tumors [30,31]. Collectively, the preclinical and clinical investigations strongly support the notion that B cell infiltrates in the tumor microenvironment not only serve as a valuable predictive biomarker, but also play a profound protective role in anti-tumor immunity [32-35].

\section{Cancer Immunotherapy}

During the last decade, great progress has been made on cancer immunotherapy including dendritic cell-based cell immunotherapy [36], chimeric antigen receptor (CAR)-T cell [37,38] and immune checkpoint blockade including CTLA-4 (Cytotoxic T-lymphocyte-associated protein 4) or PD-1 (Programmed cell death protein 1)/ PD-L1 (Programmed death-ligand 1) inhibitors [39-41]. Dendritic cells as the most professional APC possess the capability to orchestrate innate and adaptive cellular and humoral immune responses against cancer cells. Cancerantigen loaded or bioengineered dendritic cells that expressing tumor antigens have been utilized as cancer vaccines for cancer patients [42]. However dendritic cells as a tumor vaccine in clinical trials are not as effective as in preclinical animal tumor models, with the limitation of high-cost, small number and short life (2-3 days after maturation) of dendritic cells generated from peripheral blood monocytes. CAR-T cells have been successfully used to treat B-cell malignancies by targeting CD19, CD20, CD22, CD30, CD33, CD123, CD133, CD138, ROR1, $\kappa$ light chain and B-cell maturation antigen [43]. The killing of normal $\mathrm{B}$ cells besides malignant $\mathrm{B}$ cells by CAR-T cells and its serious treatment-related toxicities remains a challenge [44]. Current clinical trials reveal that CAR-T therapy have very limited efficacy on nonhematological solid tumors. Expression of regulatory molecules such as CTLA-4 and PD-1 on cytotoxic T cells has been shown to suppress the anti-tumor functions of $\mathrm{T}$ cells. Thus immune checkpoint blockade using antagonistic antibodies against the negative regulators can overcome cancer immune resistance and demonstrates promising therapeutic efficacy [45-47]. However, clinical trials showed that only partial cancer patients respond to immune checkpoint blockade $[48,49]$. B cells have multiple functions as antibody-producing cells; antigen-presenting cells, immune effector cells, and are required for adaptive $\mathrm{T}$ cell immune responses against tumors [50]. B cells also have an advantage to be easily expanded ex vivo in comparison with dendritic cells. Moreover, activated B cells can effectively present tumor lysate, antigen peptide or antigen cDNA and induce antigen-specific T cell immunoreaction against tumors [51]. Thus, B cells represent a promising approach for cancer immunotherapy, complementing the use of dendritic cells.

\section{B Cell Based Approaches for Cancer Immunotherapy}

B cells have been widely explored as a cellular adjuvant for cancer immunotherapy due to its immune-stimulatory activities. As antigen-presenting cells, B cells express CD40 and ligation with CD40 ligand on B cells robustly enhances the expression of co-stimulatory molecules CD80 and CD86 [52]. Consequently, CD40-activated B cells have potent capability to promote naïve and memory $\mathrm{T}$ activation and expansion and induce cytotoxic $\mathrm{T}$ cells immunity [53]. When pulsed with a melanoma antigen, CD40-activated B cells efficiently propel the generation of melanoma-specific T cells in vitro [54]. CD40-activated $\mathrm{B}$ cells also express adhesion molecules and chemokine receptors facilitating the cells to migrate into the secondary lymphoid organs, attract and interact with antigen-specific $\mathrm{T}$ cells [52-55]. CD40-activated B cells also function similarly to plasma cells and produce IgG [52]. In vivo, CD40-activated B cells have protective effect on various tumor models [56,57], with little toxicity to the mice [56]. Alternatively, CD40-ligated B cells loaded with tumorspecific RNA as a cancer vaccine induce tumor-specific cytotoxic $\mathrm{T}$ cell immune response, inhibit the growth of non-Hodgkin's lymphoma and improve overall survival in preclinical animal model [57]. It is interesting that leukemia B cells activated by CD40 ligation are also functionally similar to antigen-presenting cells and induce both IFN- $\gamma^{+}$ $\mathrm{CD} 4$ and cytotoxic CD8 T cell proliferating and expansion [58]. Those data together inform that CD40-activated B cells have the potential to serve as a potent cellular agent for cancer immunotherapy.

Tumor-infiltrated B cells provide another approach for B cell cancer immunotherapy. B cells infiltrated into tumor stroma function as both antigen-presenting cells and tumor antigen-specific antibody-producing cells, 
and play essential roles in anti-tumor immunity $[34,59]$. A Epstein-Barr virus immortalization in vitro assay demonstrates that primary colorectal carcinoma harbor infiltrated $\mathrm{B}$ cells that are consistent of $\mathrm{CD} 23^{+} \mathrm{CD} 80^{+}$ activated antigen-presenting cells and IgG-secreting cells. Those infiltrated B cells not only produce functional carcinoma-specific antibodies [59], but are also associated with cytolytic $\mathrm{T}$ cell response and superior prognosis in cancers $[21,22,24,29]$. Adoptive transfer of tumor-derived $\mathrm{B}$ cells further promotes anti-tumor $\mathrm{T}$ cell immunity and leads to tumor regression in preclinical breast cancer and pulmonary metastatic tumor animal models $[60,61]$. The anti-tumor property of tumor-primed B cells suggests that ex vivo expanded tumor-primed B cells could be utilized as potent $\mathrm{T}$ helper cells for cancer immunotherapy. B cells loaded with tumor-derived autophagosomes have the ability to present tumor-specific antigens selectively captured by autophagosomes and induce robust anti-tumor $\mathrm{T}$ cell response as well as antibody-mediated humoral response [62]. Administration of tumor-antigen loaded B cells as a vaccine further prevents the growth of tumors in mice [62], indicating that $\mathrm{B}$ cells activated by tumorderived autophagosomes represent a new strategy for cancer immunotherapy.

Recently, we have developed an immune-stimulatory fusion cytokine (Fusokine) named GIFT4 (Figure 1), which is a granulocyte macrophage colony-stimulating factor (GM-CSF) and common $\gamma$-chain Interleukins 4 (IL4) fusion transgene [63]. In comparison with its parental cytokines, GIFT4 fusokine gains new function distinct from its parental cytokines GM-CSF and IL-4. GIFT4 has potent capability to activate and program naive $B$ cells into immune effector cells. Programming of naïve B cells by GIFT4 fusokine involves both GM-CSF and IL-4 domains through a synergistic recruitment of GM-CSF receptor and IL-4 receptor clustered on B cell surface, which further triggers the formation of downstream signaling complex of JAK1 (The Janus kinase 1), 2, 3 and STAT1 (The signal transducer and activator of transcription 1), 3 , 5 and 6 [63]. Inhibition of JAK signaling by its specific inhibitors completely interrupted GIFT4-induced STAT1, STAT3, STAT5 and STAT6 signaling in the treated B cells and consequent B cell expansion. In contrast, combined use of parental cytokines GM-CSF and IL-4 is unable to cluster the two receptors on B cell surface and induce B cell proliferation.

Interesting, GIFT4 protein has no effect on monocytes, although GM-CSF and IL-4 together have the capability to promote monocytes differentiation into dendritic cells. GIFT4-augmented B cells (GIFT4-B cells) express co-stimulatory molecules CD40, CD80 and CD86, and produce unique immune-stimulatory cytokines, chemokines and adhesion molecules including IL- $1 \alpha$, IL-6, IL-12, GM-CSF, CCL3, CCL4 and CD54, but little IL-10 and IFN- $\gamma$ [63], apart from CD40-activated B cells [52] or innate response activator B cells [6]. With those immune properties, GIFT4-B cells function as APC-like effector cells, and consequently promote the expansion of CD314 $4^{+}$, granzyme B-, granulysin- and IFN-producing cytotoxic $\mathrm{T}$ cells that selectively kill human melanoma cells both in vitro and in vivo [63]. Moreover, GIFT4 fusokine induces B cell-dependent anti-tumor immunity in murine melanoma models [63], involving both APC-like B effector cells and GM-CSFproducing innate response activator B-cells $[6,63]$. In our investigation of GIFT4 as a potential vaccine adjuvant, we also discovered that GIFT4-coated virus-like particles enhance anti-HIV antigen-specific antibody production in vivo [64], suggesting additional effect of GIFT4 on the antibody-secreting cells. Indeed, we have found that administration of GIFT4 protein induces robust anti-melanoma specific-antibody production in murine melanoma model (Unpublished data). We have further extended our investigation to human chronic lymphocytic leukemic (CLL) B cells, and examined the immune activity of GIFT4-stimulated CLL B cells (GIFT4-CLL cells). Unlike CD40-activated CLL cells [58], TLR9 ligand-treated CLL cells [65] or normal GIFT4-B cells [63], GIFT4-CLL cells produce immune-stimulatory cytokines including IL-1 $\beta$, IL-2, IL-6, IL-8, ICAM-1 and prime autologous $\mathrm{T}$ cells to proliferate, express tumorkilling molecules IFN- $\gamma, \mathrm{CD} 314$, perforin and granzyme $\mathrm{B}$, and lyse autologous primary leukemic cells [66]. Taken together, GIFT4 induces broad anti-tumor B cell immune

$B$

MWLQSLLLLGTVACSISAPARSPSPSTQPWEHV NAIQEARRLLNLSRDTAAEMNETVEVISEMFDLQ EPTCLQTRLELYKQGLRGSLTKLKGPLTMMASH YKQHCPPTPETSCATQTITFESFKENLKDFLLVIP FDCWEPVQESMGLTSQLLPPLFFLLACAGNFVH GHKCDITLQEIIKTLNSLTEQKTLCTELTVTDIFAA SKNTTEKETFCRAATVLRQFYSHHEKDTRCLGA TAQQFHRHKQLIRFLKRLDRNLWGLAGLNSCPV KEANQSTLENFLERLKTIMREKYSKCSS

Figure 1. Structure of GIFT4 protein. (A) GIFT4 protein structure that contains GM-CSF and IL-4 domains. (B) Amino acids of human GIFT4 
responses either through GIFT4-programmed B effector cells that further prime tumor-killing cytotoxic $\mathrm{T}$ cell response, or through the augmentation of tumor-specific antibody production. Those results provide a strong basis for the potential utilization of GIFT4 fusokine and GIFT4augmented B cells as well as GIFT4-converted CLL cells for cancer immunotherapy in human.

\section{Conclusion}

B cells play pivotal roles in immune defense system, which bridge the innate and the adaptive immunities against cancers. Augmented B cells including GIFT4-B cells and expanded tumor-infiltrated B cells have potent immunestimulatory activities and anti-tumor function by either priming cytotoxic $\mathrm{T}$ cell response or producing anti-tumor specific antibodies. We predict that GIFT4 and GIFT4augmented B cells as potential immune therapeutics could provide a new approach for cancer immunotherapy.

\section{Acknowledgement}

This work was supported by the Winship Robbins Scholar Award, the Winship Melanoma Research Fund and the Developmental Fund of the Winship Cancer Center Support Grant (5P30CA138292-06) (to JD).

\section{Disclosure}

The authors declare that they have no competing interest.

\section{References}

1. Cooper MD. The early history of B cells. Nature reviews Immunology 2015; 15: 191-197.

2. Borhis G, Richard Y. Subversion of the B-cell compartment during parasitic, bacterial and viral infections. BMC Immunology 2015, 16: 15.

3. Khan WN, Wright JA, Kleiman E, et al. B-lymphocyte tolerance and effector function in immunity and autoimmunity. Immunologic Research 2013; 57: 335-353.

4. Popi AF, Longo-Maugeri IM, Mariano M. An overview of B-1 cells as antigen-presenting cells. Frontiers in Immunology 2016; 7: 138.

5. Bao Y, Cao X. The immune potential and immunopathology of cytokine-producing B cell subsets: A comprehensive review. Journal of Autoimmunity 2014; 55: 10-23.

6. Rauch PJ, Chudnovskiy A, Robbins CS, et al. Innate response activator B cells protect against microbial sepsis. Science 2012; 335: 597-601.

7. Zasada M, Rutkowska-Zapala M, Lenart M, et al. The role of IRA B cells in selected inflammatory processes. Postepy higieny i medycyny doswiadczalnej 2016; 70: 194-199.

8. Rosser EC, Mauri C. Regulatory B cells: Origin, phenotype and function. Immunity 2015; 42: 607-612.

9. Wang JZ, Zhang YH, Guo XH, et al. The double-edge role of B cells in mediating antitumor T-cell immunity: Pharmacological strategies for cancer immunotherapy. International Immunopharmacology 2016; 36: 73-85.
10. Garnelo M, Tan A, Her Z, et al. Interaction between tumourinfiltrating $\mathrm{B}$ cells and $\mathrm{T}$ cells controls the progression of hepatocellular carcinoma. Gut 2015.

11. Krishnan S, Bakker E, Lee C, et al. Successful combined intratumoral immunotherapy of established murine mesotheliomas require B-cell involvement. Journal of interferon \& cytokine research: The Official Journal of the International Society for Interferon and Cytokine Research 2015; 35: 100-107.

12. Lao XM, Liang YJ, Su YX, et al. Distribution and significance of interstitial fibrosis and stroma-infiltrating B cells in tongue squamous cell carcinoma. Oncology Letters 2016; 11: 2027-2034.

13. Zhang Y, Gallastegui N, Rosenblatt JD. Regulatory B cells in anti-tumor immunity. International Immunology 2015; 27: $521-530$

14. Tang A, Dadaglio G, Oberkampf M, et al. B cells promote tumor progression in a mouse model of HPV-mediated cervical cancer. International Journal of Cancer 2016.

15. Hiraoka N, Ino Y, Yamazaki-Itoh R, et al. Intratumoral tertiary lymphoid organ is a favourable prognosticator in patients with pancreatic cancer. British Journal of Cancer 2015; 112: 1782-1790.

16. Castino GF, Cortese N, Capretti G, et al. Spatial distribution of $\mathrm{B}$ cells predicts prognosis in human pancreatic adenocarcinoma. Oncoimmunology 2016; 5: e1085147.

17. Pichler R, Fritz J, Zavadil C, et al. Tumor-infiltrating immune cell subpopulations influence the oncologic outcome after intravesical bacillus calmette-guerin therapy in bladder cancer. Oncotarget 2016.

18. Goc J, Fridman WH, Sautes-Fridman C, et al. Characteristics of tertiary lymphoid structures in primary cancers. Oncoimmunology 2013; 2: e26836.

19. Hennequin A, Derangere V, Boidot R, et al. Tumor infiltration by Tbet + effector $T$ cells and CD20+ B cells is associated with survival in gastric cancer patients. Oncoimmunology 2016; 5: e1054598.

20. Ladanyi A. Prognostic and predictive significance of immune cells infiltrating cutaneous melanoma. Pigment Cell \& Melanoma Research 2015; 28: 490-500.

21. Garg K, Maurer M, Griss J, et al. Tumor associated B cells in cutaneous primary melanoma and improved clinical outcome. Human pathology 2016.

22. Fremd C, Stefanovic S, Beckhove P, et al. Mucin 1-specific B cell immune responses and their impact on overall survival in breast cancer patients. Oncoimmunology 2016; 5: e1057387.

23. Lundgren S, Berntsson J, Nodin B, et al. Prognostic impact of tumour-associated B cells and plasma cells in epithelial ovarian cancer. Journal of Ovarian Research 2016; 9: 21.

24. Berntsson J, Nodin B, Eberhard J, et al. Prognostic impact of tumour-infiltrating B cells and plasma cells in colorectal cancer. International Journal of Cancer 2016; 139: 11291139.

25. Nielsen JS, Sahota RA, Milne K, et al. CD20+ tumor- 
infiltrating lymphocytes have an atypical CD27-memory phenotype and together with CD8+ T cells promote favorable prognosis in ovarian cancer. Clinical Cancer Research: An Official JAACR 2012; 18: 3281-3292.

26. Nielsen JS, Nelson BH. Tumor-infiltrating B cells and T cells: Working together to promote patient survival. Oncoimmunology 2012; 1: 1623-1625.

27. Lohr M, Edlund K, Botling J, et al The prognostic relevance of tumour-infiltrating plasma cells and immunoglobulin kappa $\mathrm{C}$ indicates an important role of the humoral immune response in non-small cell lung cancer. Cancer Letters 2013; 333: $222-228$.

28. Yokoyama S, Higashi M, Kitamoto S, et al. Aberrant methylation of $\mathrm{MUC1}$ and MUC4 promoters are potential prognostic biomarkers for pancreatic ductal adenocarcinomas. Oncotarget 2016.

29. Kroeger DR, Milne K, Nelson BH. Tumor-infiltrating plasma cells are associated with tertiary lymphoid structures, cytolytic T-cell responses and superior prognosis in ovarian cancer. Clinical cancer research. Ameri Asso Can Res 2016; 22: 3005-3015.

30. Campa MJ, Moody MA, Zhang R, et al. Interrogation of individual intratumoral B lymphocytes from lung cancer patients for molecular target discovery. Cancer Immunology, Immunotherapy 2016; 65: 171-180.

31. Quan H, Fang L, Pan H, et al An adaptive immune response driven by mature, antigen-experienced $\mathrm{T}$ and $\mathrm{B}$ cells within the microenvironment of oral squamous cell carcinoma. International Journal of Cancer 2016; 138: 2952-2962.

32. Bremnes RM, Busund LT, Kilvaer TL, et al. The role of tumor-infiltrating lymphocytes in development, progression and prognosis of non-small cell lung cancer. Journal of Thoracic Oncology. International Association for the Study of Lung Cancer 2016; 11: 789-800.

33. Dieu-Nosjean MC, Giraldo NA, Kaplon H, et al. Tertiary lymphoid structures, drivers of the anti-tumor responses in human cancers. Immunological Reviews 2016; 271: 260275.

34. Germain C, Gnjatic S, Dieu-Nosjean MC. Tertiary lymphoid structure-associated B cells are key players in anti-tumor immunity. Frontiers in Immunology 2015; 6: 67.

35. Muenst S, Laubli H, Soysal SD, et al. The immune system and cancer evasion strategies: Therapeutic concepts. Journal of Internal Medicine 2016; 279: 541-562.

36. Ni M, Hoffmann JM, Schmitt M, et al. Progress of dendritic cell-based cancer vaccines for patients with hematological malignancies. Expert Opinion on Biological Therapy 2016; $1-11$.

37. Haji-Fatahaliha M, Hosseini M, Akbarian A, et al. CARmodified T-cell therapy for cancer: An updated review. Artificial Cells, Nanomedicine and Biotechnology 2015; $1-11$.

38. Zhang H, Ye ZL, Yuan ZG, et al. New strategies for the treatment of solid tumors with CAR-T cells. International Journal of biological sciences 2016; 12: 718-729.
39. Kyi C, Postow MA. Immune checkpoint inhibitor combinations in solid tumors: opportunities and challenges. Immunotherapy 2016; 8: 821-837.

40. Adachi K, Tamada K. Immune checkpoint blockade opens an avenue of cancer immunotherapy with a potent clinical efficacy. Cancer science 2015; 106: 945-950.

41. Lee CS, Cragg M, Glennie M, Johnson P. Novel antibodies targeting immune regulatory checkpoints for cancer therapy. British journal of clinical pharmacology 2013; 76: 233-247.

42. Palucka K, Banchereau J. Dendritic-cell-based therapeutic cancer vaccines. Immunity 2013; 39: 38-48.

43. Almasbak H, Aarvak T, Vemuri MC. CAR T cell Therapy: A Game Changer in Cancer Treatment. Journal of immunology research 2016; 2016: 5474602.

44. Brudno JN, Kochenderfer JN. Toxicities of chimeric antigen receptor T cells: recognition and management. Blood 2016.

45. Snyder A, Makarov V, Merghoub T, Yuan J, Zaretsky JM, et al. Genetic basis for clinical response to CTLA-4 blockade in melanoma. The New England journal of medicine 2014; 371: 2189-2199.

46. Topalian SL, Hodi FS, Brahmer JR, Gettinger SN, Smith DC, et al. Safety, activity, and immune correlates of anti-PD-1 antibody in cancer. The New England journal of medicine 2012; 366: 2443-2454.

47. Brahmer JR, Tykodi SS, Chow LQ, Hwu WJ, Topalian SL, et al. Safety and activity of anti-PD-L1 antibody in patients with advanced cancer. The New England journal of medicine 2012; 366: 2455-2465.

48. Wargo JA, Cooper ZA, Flaherty KT. Universes collide: combining immunotherapy with targeted therapy for cancer. Cancer discovery 2014; 4: 1377-1386.

49. Robert L, Ribas A, Hu-Lieskovan S. Combining targeted therapy with immunotherapy. Can $1+1$ equal more than 2 ? Seminars in immunology 2016; 28: 73-80.

50. DiLillo DJ, Yanaba K, Tedder TF. B cells are required for optimal CD4+ and CD8+ T cell tumor immunity: therapeutic $\mathrm{B}$ cell depletion enhances B16 melanoma growth in mice. Journal of immunology 2010; 184: 4006-4016.

51. Guo S, Xu J, Denning W, Hel Z. Induction of protective cytotoxic T-cell responses by a B-cell-based cellular vaccine requires stable expression of antigen. Gene therapy 2009; 16: $1300-1313$.

52. Wennhold K, Shimabukuro-Vornhagen A, Theurich S, von Bergwelt-Baildon M. CD40-activated B cells as antigenpresenting cells: the final sprint toward clinical application. Expert review of vaccines 2013; 12: 631-637.

53. Gonzalez NK, Wennhold K, Balkow S, Kondo E, Bolck B, et al. Imaging of initial B-T-cell interactions in the setting of B-cell based cancer immunotherapy. Oncoimmunology 2015; 4: e1038684.

54. Lapointe R, Bellemare-Pelletier A, Housseau F, et al. CD40stimulated B lymphocytes pulsed with tumor antigens are effective antigen-presenting cells that can generate specific T cells. Cancer research 2003; 63: 2836-2843. 
55. von Bergwelt-Baildon M, Shimabukuro-Vornhagen A, Popov A, et al. CD40-activated B cells express full lymph node homing triad and induce T-cell chemotaxis: Potential as cellular adjuvants. Blood 2006; 107: 2786-2789.

56. Wennhold K, Weber TM, Thelen M, et al. CD40-activated B cells induce anti-tumor immunity in vivo. Oncotarget 2016.

57. Sorenmo KU, Krick E, Coughlin CM, et al. CD40-activated $\mathrm{B}$ cell cancer vaccine improves second clinical remission and survival in privately owned dogs with non-Hodgkin's lymphoma. PloS One 2011; 6: e24167.

58. Buhmann R, Nolte A, Westhaus D, et al. CD40-activated B-cell chronic lymphocytic leukemia cells for tumor immunotherapy: stimulation of allogeneic versus autologous $\mathrm{T}$ cells generates different types of effector cells. Blood 1999; 93: 1992-2002.

59. Maletzki C, Jahnke A, Ostwald C, et al. Ex vivo clonally expanded B lymphocytes infiltrating colorectal carcinoma is of mature immunophenotype and produce functional IgG. PloS One 2012; 7: e32639.

60. Li Q, Lao X, Pan Q, et al. Adoptive transfer of tumor reactive $\mathrm{B}$ cells confers host T-cell immunity and tumor regression. Clinical Cancer Research: An Official Journal of the American Association for Cancer Research 2011; 17: 4987-4995.
61. Li Q, Teitz-Tennenbaum S, Donald EJ, et al. In vivo sensitized and in vitro activated $\mathrm{B}$ cells mediate tumor regression in cancer adoptive immunotherapy. Journal of Immunology 2009; 183: 3195-3203.

62. Ren H, Zhao S, Li W, et al. Therapeutic antitumor efficacy of B cells loaded with tumor-derived autophagasomes vaccine (DRibbles). Journal of Immunotherapy 2014; 37 : 383-393.

63. Deng J, Yuan S, Pennati A, et al. Engineered fusokine GIFT4 licenses the ability of B cells to trigger a tumoricidal T-cell response. Cancer Research 2014; 74: 4133-4144.

64. Feng H, Zhang H, Deng J, et al. Incorporation of a GPIanchored engineered cytokine as a molecular adjuvant enhances the immunogenicity of HIV VLPs. Scientific Reports 2015; 5: 11856.

65. Ghamlouch H, Ouled-Haddou H, Guyart A. TLR9 Ligand (CpG Oligodeoxynucleotide) induces CLL B-cells to differentiate into CD20(+) antibody-secreting cells. Frontiers in Immunology 2014; 5: 292.

66. Deng J, Pennati A, Cohen JB, et al. GIFT4 fusokine converts leukemic B cells into immune helper cells. Journal of translational medicine 2016; 14: 106.

\section{Correspondence to:}

Jiusheng Deng,

Department of Hematology and Medical Oncology,

Winship Cancer Institute,

Emory University,

1365B Clifton Road,

Atlanta, GA,

USA.

Tel: 4047781763

E-mail: jdeng2@emory.edu 